\title{
Author Correction: PBX3 is targeted by multiple miRNAs and is essential for liver tumour-initiating cells
}

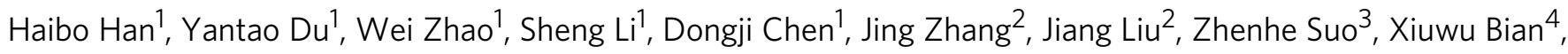
Baocai Xing ${ }^{5} \&$ Zhiqian Zhang ${ }^{1}$

Correction to: Nature Communications https://doi.org/10.1038/ncomms9271, published online 30 September 2015

This Article contains an error in Figure 3. In panel g, images representing miR-222-TuD and miR-424-TuD were both taken from the miR-222-TuD image. The correct version of Figure 3 is shown below as Fig. 1.

Published online: 21 May 2019

\footnotetext{
${ }^{1}$ Department of Cell Biology, Key Laboratory of Carcinogenesis and Translational Research (Ministry of Education/Beijing), Center for Molecular and Translational Medicine, Peking University Cancer Hospital and Institute, 52 Fucheng Road, Beijing 100142, China. ${ }^{2}$ Beijing Institute of Genomics, Chinese Academy of Sciences, Beijing 100101, China. ${ }^{3}$ Department of Pathology, Oslo University Hospital, Institute of Clinical Medicine, Faculty of Medicine, University of Oslo, Oslo 0379, Norway. ${ }^{4}$ Institute of Pathology and Southwest Cancer Center, Southwest Hospital, Third Military Medical University, Chongqing 400038, China. ${ }^{5}$ Department of Hepatobiliary Surgery I, Peking University Cancer Hospital and Institute, Beijing 100142, China. Correspondence and requests for materials should be addressed to Z.Z. (email: zlzqzhang@bjmu.edu.cn)
} 
a

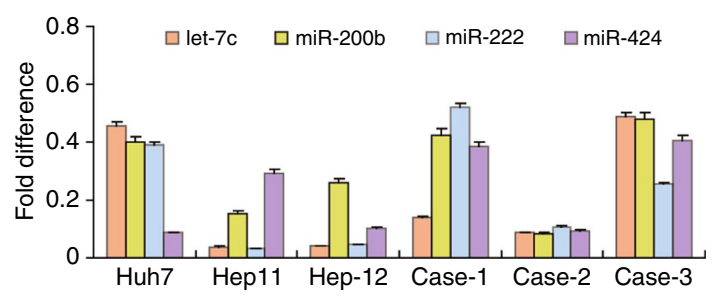

C

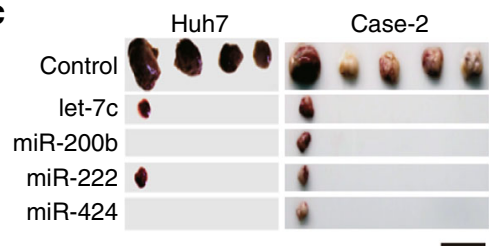

d

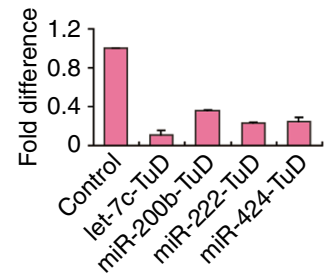

f

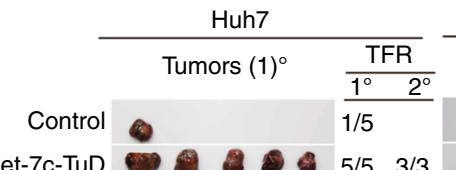

let-7c-TuD $5 / 5 \quad 3 / 3$ miR-200b-TuD $5 / 5 \quad 3 / 3$

miR-222-TuD $5 / 5 \quad 3 / 3$

miR-424-TuD $4 / 5 \quad 3 / 3$

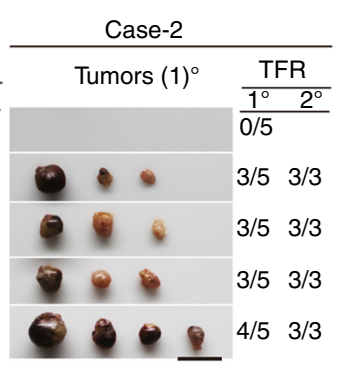

g

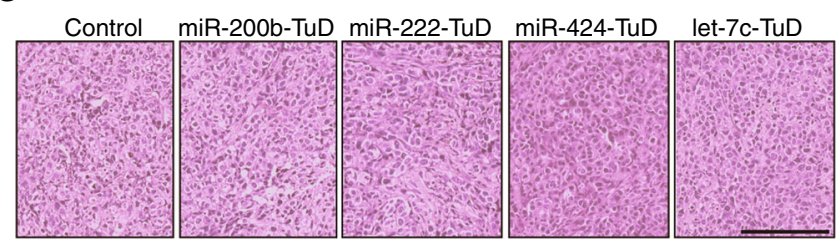

e
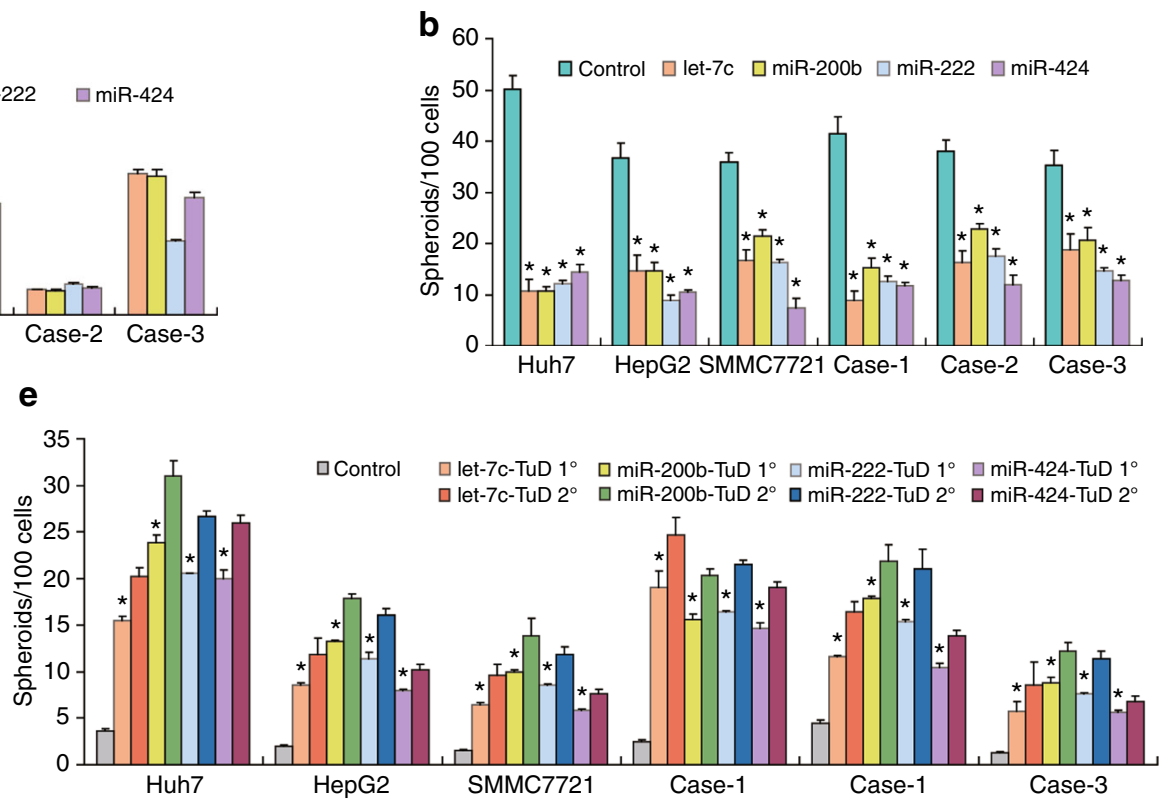

h
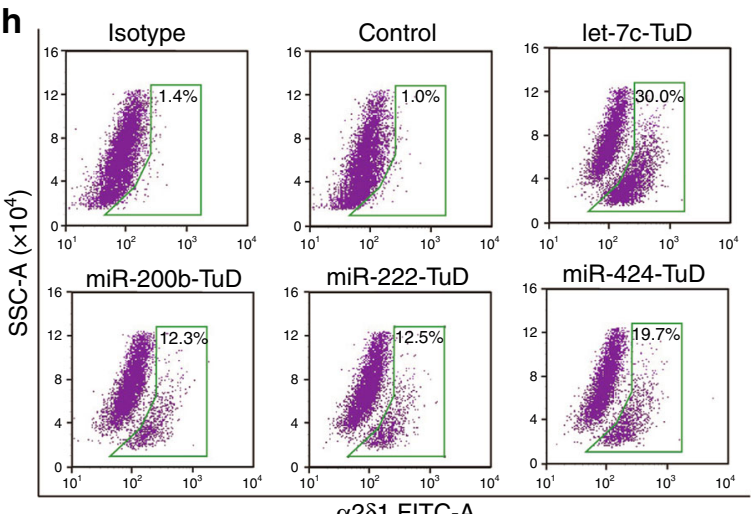

i

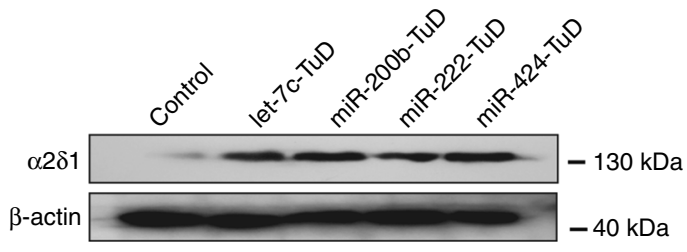

j

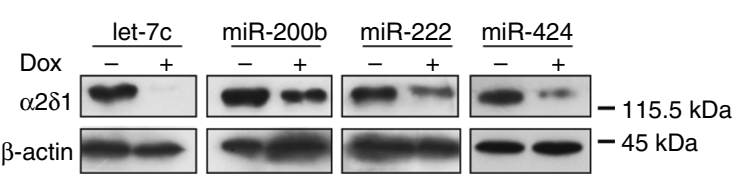

Fig. 1 